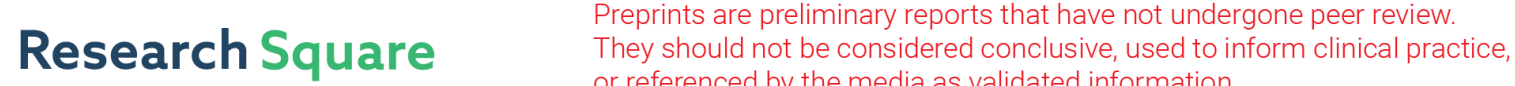 \\ or referenced by the media as validated information. \\ MRI Modality-based Brain Tumor Segmentation Using Deep Neural Networks
}

\section{Pankaj Eknath Kasar ( $\nabla$ pankaj@dbatu.ac.in )}

Dr Babasaheb Ambedkar Technological University https://orcid.org/0000-0001-7548-7608

\section{Shivajirao M. Jadhav}

Dr Babasaheb Ambedkar Technological University

\section{Vineet Kansal}

Institute of Engineering and Technology

\section{Research}

Keywords: UNET, SEGNET, Deep Neural Networks, Brain tumor, segmentation, Magnetic Resonance Imaging (MRI)

Posted Date: May 12th, 2021

DOI: https://doi.org/10.21203/rs.3.rs-496162/v1

License: (9) This work is licensed under a Creative Commons Attribution 4.0 International License. Read Full License 


\title{
MRI modality-based Brain Tumor Segmentation using Deep Neural Networks
}

\author{
Pankaj E. Kasar ${ }^{1 *}$, Shivajirao M. Jadhav ${ }^{2}$, Vineet Kansal ${ }^{3}$ \\ ${ }^{1,2}$ Dr. Babashaeb Ambedkar Technological University, Lonere, Raigad, India, ${ }^{3}$ Institute of Engineering \& Technology, \\ Lucknow, U.P., India \\ erpankajkasar@rediffmail.com, smjadhav@dbatu.ac.in, vineetkansal@ietlucknow.ac.in
}

\begin{abstract}
The tumor detection is major challenging task in brain tumor quantitative evaluation. In recent years, owing to non-invasive and strong soft tissue comparison, Magnetic Resonance Imaging (MRI) has gained great interest. MRI is a commonly used image modality technique to locate brain tumors. An immense amount of data is produced by the MRI. Heterogeneity, isointense and hypointense tumor properties restrict manual segmentation in a fair period of time, thus restricting the use of reliable quantitative measures in clinical practice. In the clinical practice manual segmentation task is quite time consuming and their performance is highly depended on the operator's experience. Accurate and automated tumor segmentation techniques are also needed; however, the severe spatial and structural heterogeneity of brain tumors makes automatic segmentation a difficult job. This paper proposes fully automatic segmentation of brain tumors using encoderdecoder based convolutional neural networks. The paper focuses on well-known semantic segmentation deep neural networks i.e., UNET and SEGNET for segmenting tumors from Brain MRI images. The networks are trained and tested using freely accessible standard dataset, with Dice Similarity Coefficient (DSC) as metric for whole predicted image i.e., including tumor and background. UNET's average DSC on test dataset is 0.76 whereas for SEGNET we got average DSC 0.67. The evaluation of results proves that UNET is having better performance than SEGNET.
\end{abstract}

Keywords - UNET, SEGNET, Deep Neural Networks, Brain tumor, segmentation, Magnetic Resonance Imaging (MRI).

\section{INTRODUCTION}

Now days deep learning techniques are promising exciting solutions in many research fields like Natural Language Processing, Image analysis and various Expert Systems. Notable progress has been achieved in Medical imaging field in past few years due to advancement in CNNs. It also seen as key method for different applications in near future. Segmentation of abnormality within medical imaging is one of the prime challenges like brain tumor segmentation [1], cardiac ventricle segmentation [2], abdominal organ segmentation [3], and cell segmentation in biological images [4].
In recent years, human knowledge, with advances in the field of health care, has shown diseases to have stumbled, but even cancer, because of its uneven existence, remains a curse for humans. Brain tumor cancer is considered to be one of the deadly diseases. Brain is central and administrative center in our human body. It is responsible for carrying out all tasks such as breathing, the activity of muscles, our senses in the human body through a massive network of internally connected neurons. Brain tumors are caused by irregular cell development in the brain [5], which affects the operation of the nervous system, resulting in a very low life span at the highest degree. The diagnosis of early-stage brain tumors relies on the expertise and level of competence of the physicians, making it easier for the patient to heal and expect life to continue. Automated segmentation of brain tumors is an efficient method to help clinicians pursue a good course of action. This automatic segmentation using deep neural networks also allows physicians with little knowledge to make the right decisions and experienced physicians to solve difficult cases. This method incorporates images captured by magnetic resonance (MR) imaging instruments that are used extensively by brain diagnostic radiologists.

Brain tumors are typically divided into two groups, i.e., benign and malignant tumors. Benign tumors develop steadily, originating in the brain only suggests that no tumor in the body will propagate this kind. so it is presumed that it is non-cancerous (non-progressive) and less aggressive. On the opposite, the type of malignant tumor is a harmful tumor, expanding rapidly with unknown borders that invade other strong body cells. It is recognized as a primary malignant tumor if such a tumor is found in the brain. It is a secondary malignant tumor [6] if it originates somewhere in the body and extends to the brain.

However other general forms of brain tumors are glioma, meningioma and pituitary tumors. The most prominent noncancerous tumors that exist in the thin membranes covering the spinal cord and the brain are meningiomas. Tumors that develop inside the material of the brain [7] are a series of gliomas. With a minimum lifespan of nearly a few years, high-grade gliomas are one of the most aggressive brain tumors. In the brain's pituitary gland, pituitary tumors are 
formed. The uniform shape and inherent essence of all these tumors are shown in Fig. 1.

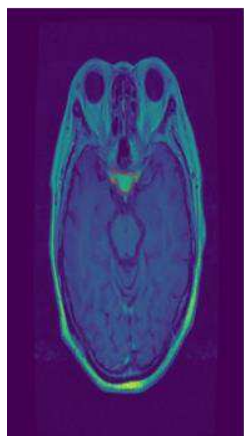

(a)

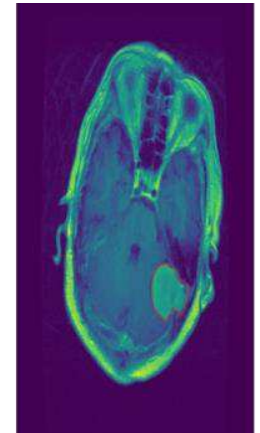

(b)

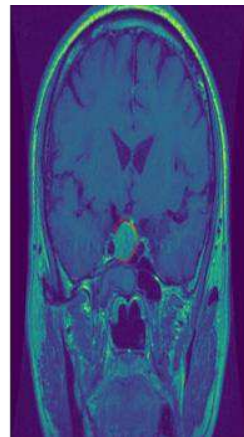

(c)
Fig. 1 Illustrations of three typical brain tumors: (a) meningioma; (b) glioma; and (c) pituitary tumor. Red lines indicate the tumor border

Normally, main tumors such as meningioma (MEN), astrocytoma (AS), medulloblastoma (MED), glioblastoma multiforme (GBM), and secondary metastasis tumors (MET) are typically identified using T1-weighted contrast-enhanced imaging techniques. Via a stimulation of $0.150 .20 \mathrm{mMol} / \mathrm{kg}$ contrast content (Gadolinium) in the patients, these tumors are slightly better visualized with T1-weighted contrastenhanced images [8].

Traditional algorithms rely heavily on a manual extraction of features which require greater expertise. But feature extraction in deep learning, on the other hand, is automatic. Each layer of the neural network (i.e. deep network with many hidden layers) uses features of the previous layer in order to learn higher-level features. This paper therefore introduces two state-of-the-art UNET and SEGNET segmentation networks for segment anomalies in MRI images. Since these networks offer an end-to-end approach to a problem, they have stringent computing capacity criteria for the large scale of the dataset. Generally, graphics processing units (GPUs) are used to satisfy this computational criterion and thus facilitate network training within a suitable period of time.

The remaining of the paper is organized as follows. Section 2 literature review of the existing work on Brain tumor Segmentation. Section 3.1 describes the detailed architecture of semantic segmentation deep neural networks 3.1.a UNET and 3.1.b. SEGNET. The standard dataset used for training and testing is publicly available is enumerated in Section 3.2. Different data augmentation operations and preprocessing steps applied before training the networks are demonstrated in section 3.3. The hardware and software resources used for training the network are mentioned in Section 4. The metrics used to measure the performance of network with loss function is enumerated in Section 4.1. The dice similarity coefficient, precision, accuracy obtained during training with loss convergence are specified in Section 4.2. The segmentation results of UNET and SEGNET are presented in section 4.3. Finally, the discussion along with conclusion is drawn in section 5 with future improvements.

\section{RELATED WORK}

\subsection{Manual Segmentation}

The tumor borders and interest structures are manually drawn or painted with various labels in anatomical structures [9]. In manual segmentation, human experts not only make use of the details provided in the image, but also make use of additional expertise such as brain anatomy. This manual segmentation is mostly error-prone as the structural and spatial variety of the tumor portion.

\subsection{Fully automatic segmentation}

Now days, the several studies have been proposed different neural network based automated systems for detecting and segmenting brain tumors using MR images. The various fully automatic brain tumor segmentation techniques have been discussed as follows: -

Sergio Pereira et al., (2016) [10] suggested a novel segmentation neural network. Their assumption is that small size kernels allows the deep architecture to avoid overfitting by assigning the fewer number of weights in the network. They also proved that preprocessing step of pixel intensity normalization along with data augmentation is very effective for brain tumor segmentation in BRATS 2013 MRI images. The author also participated in BRATS 2015 Challenge and secured second position.

Dong et al., (2017) [11] proposed a fully automatic brain tumor detection and segmentation method using UNET based deep neural network. They show that their method can provide efficient and robust segmentation on MRI brain tumor images from BRATS 2015 benchmark dataset.

Mohammad Havaei et al., (2017) [12] illustrated a CNN based different segmentation architectures like Two-pathway architecture and Cascaded architectures for segmenting brain MRI images form BRATS 2013 datasets. Their proposed system exploits both local and global contextual features simultaneously. The final layer is a convolutional implementation of a fully connected layer which allows a 40-fold speed up. Results evaluated on the 2013 BRATS test dataset exposed that their network proved best performance over the published state-of-the-art networks at that time.

Saddam Hussain et al., (2017) [13] proposed CNN architecture for brain tumor segmentation which incorporates both global and local features maps. The use of max-pooling and drop out layers stabilize the testing process, improving the training and test speed by decreasing features in a completely connected layer and reducing the number of parameters and the probability of over-fitting. Here also pixel normalization as preprocessing step is used. 
The postprocessing step applied in which minor false positives are picked-out using morphological operations. The research work is carried out on BRATS 2013 brain MRI dataset.

Wang et al., (2017) [14] recommended that the usage of a fully convolutional neural network for automated medical image segmentation did not show adequately reliable and stable results for clinical use as did the lack of imagespecific adaptation and the lack of generalization to previously unseen object classes. To overcome these issues, they proposed a new dynamic segmentation architecture for deep learning, including CNNs in the bounding box and scripting pipeline. They were also suggesting image contextual fine tuning to fit the $\mathrm{CNN}$ model to a different image that could either be unattended or without extra user experiences (supervised). They have used BRATS 2015 data set for their experimentation.

Konstantinos et al., (2017) [15] implemented a dual pathway, DeepMadic, 3D CNN architecture for automatic lesion segmentation that outperform state-of-the-art on challenging data like BRATS 2015 and ISLES 2015. They also proved the benefits of using small convolutional kernels in 3D CNNs, thereby reducing the trainable parameters with computational cost which allowed to develop a deeper discriminative network. They also proposed an efficient solution for processing large image context by the use of parallel convolutional pathways for multi-scale processing.

Liang Chen et al., 2017[16] suggested a new deep neural network called Dense-Res-Inception Net (DRINet). Features acquired by standard convolution layers are not ideal when various characteristics such as strength, location, form, and tumor size shift. DRINet solves this dilemma. It consists of three blocks, i.e. a convolution block with dense links, a deconvolution block with residual inception modules, and a unpooling block. The proposed architecture surpasses the UNet in three separate difficult applications, namely multiclass cerebrospinal fluid segmentation (CSF) on brain CT images, multi-organ segmentation on abdominal CT images, multi-class brain tumor segmentation on MR images.

Dmitry Lachinov et al., 2018 [17], presented automatic brain tumor segmentation algorithm which solves following two problems arises when you use multimodal MRI scans, i.e Complex input and overfitting of classifier. The problem of heterogeneous input is solved by using multiple encoders UNET sothat every individual input modality produces corresponding features maps separately and then the way to combine these feature maps is also described by author. Moreover, they also presented the way to efficiently fuse multiple models operating on the different resolution that forms a cascade of classifiers. Each succeeding classifier takes predictions of previous one and improves the segmentation for its specific size. This allows iterative result refinements with a smaller number of parameters than in corresponding deep networks. Author have used BRATS 2018 brain MRI images and mean Dice score of 0.908 , $0.784,0.844$ was concluded on validation dataset for the Whole tumor, Enhancing tumor and Tumor core respectively.

Cui et al., (2018) [18] have developed a novel, fully automated segmentation system based on MRI data comprising in vivo brain gliomas. Their method not only localizes the tumor area, but can also specifically segment the intratumor structure. The proposed deep cascaded CNN consists of two sub-networks: (1) a tumor localization network (TLN) and (2) an intratumor classification network (ITCN). The goal of the first subnetwork was to identify the area of the tumor from the MRI. The ITCN was then used to mark the identified tumor area into multiple sub-regions. The proposed method was validated for multimodal brain tumor segmentation (BRATS 2015) data sets showing positive segmentation outcomes and faster segmentation speed.

Hai Thanh Le et al., 2020 [19] presented U-NET based deep neural network to categorize tumorous tissues into four classes for necrosis, edema, non-enhancing and enhancing tumor. Their dataset used for experimentation was the Brain Tumor Segmentation Challenge 2013 (BRATS 2013) dataset. They have measured the best performance score with the metric values Dice and sensitivity of 0.83 and 0.85 respectively.

Mostefa Ben Naceur et al., 2020 [20] developed 3 staged pipeline to enhance the prediction of Glioblastomas (GBM) tumoral regions. They developed deeper Convolutional Neural Networks (CNNs) in the first stage, then extracted multi-dimensional features from higher-resolution representation of CNNs in the second stage, where the extracted characteristics of CNNs are fed into various traditional machine learning algorithms for example Random Forest (RF), Logistic Regression (LR) and principal component analysis (PCA-SVM). They have worked on BRATS-2019 dataset. The average Dice score of their pipeline got $0.85,0.76$, and 0.74 for whole tumor, tumor core, and enhancing tumor, respectively.

\section{BACKGROUND AND METHODOLOGY}

\subsection{Semantic Segmentation}

The ImageNet classification challenge are outperformed by CNN based classifier networks such as AlexNet [21], VGGNet [22], and GoogLeNet [23] etc. This networks are now configured by classifying pixels to accomplish the image segmentation task. However, the performances of above networks are not equally good, but the concept of down-sampling up-sampling using pooling and unpooling layers makes the segmentation task comparatively good. In Semantic segmentation, the image is segmented on a pixellabel basis, is process of linking each pixel in an image to a class label. These labels can be a person, car, flower, piece 
of furniture, tumor etc. Some of its primary applications are in autonomous vehicles, human-computer interaction, robotics, and photo editing/creativity tools etc. In this, the deep neural networks are trained at end to end, pixel to pixel manner on each layer. Following section will illustrates two semantic segmentation neural networks like UNET and SEGNET.

\section{1. a. UNET:}

The UNET network architecture is illustrated in Fig 2. This is very popular semantic segmentation network for biomedical imaging proposed by Olaf Ronneberger in May 2015 [24]. It consists of three parts i) The contracting/downsampling path ii) Bottleneck iii) The expanding/upsampling path. This architecture is considered as extension of Fully Convolutional Network [25] in a way that, i) UNET is symmetric, ii) the skip connections between the contracting path and the expanding path apply a concatenation operator instead of a sum.

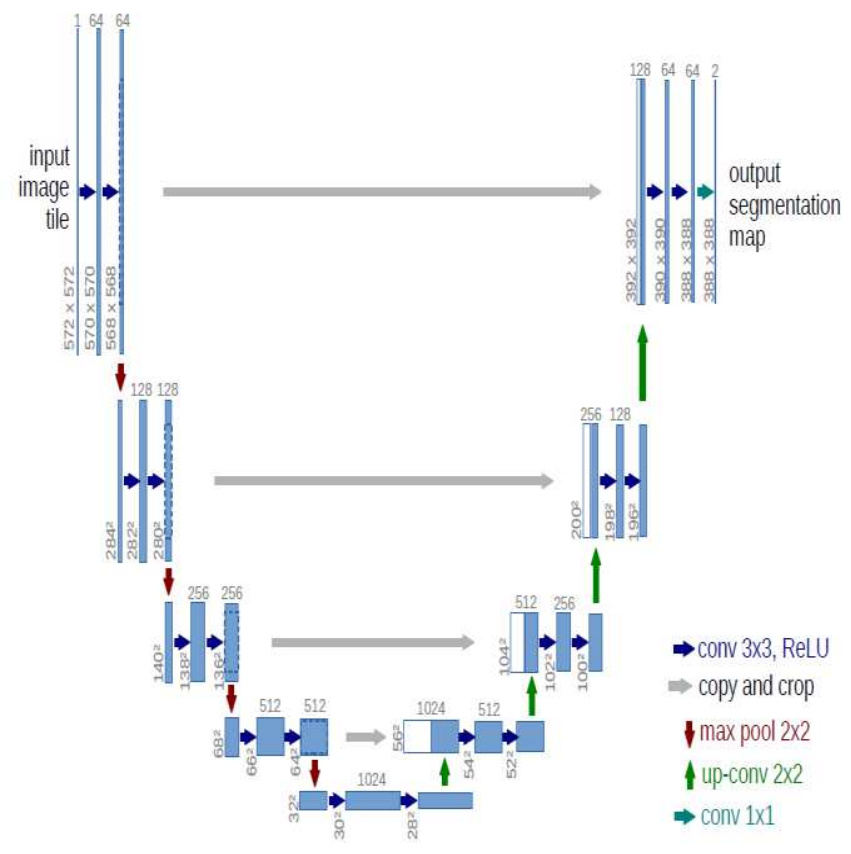

Fig. 2. The architecture of the U-net. The boxes correspond to a map of a multi-channel function. The number of channels is displayed at the top of the box. The x-y-size is seen at the bottom left edge of the box. White boxes reflect copied feature maps. The arrows signify the various operations [24].

The skip connections are responsible for proving local information to global information while upsampling. Due to its symmetric nature, the upsampling path have large number of feature maps, which allows to transfer information.

Contract/downsampling path: consists of 4 blocks, each of which consists of two $3 \times 3$ Convolution Layer + Activation function, followed by a $2 \times 2$ Max Pooling layer. Notice that the number of feature maps doubles at each pooling layer, starting with 64 feature maps for the first block, 128 for the second block, and so on. The aim of this contracting path is to collect the context of the input image and via skip connections, this rough contextual information will then be passed to the upsampling path.

Bottleneck: This section of the network is the juncture between contracting and expanding routes. It's constructed from only two convolution layers (with batch normalization).

Expanding/upsampling path: It is also collection of 4 blocks with each block consists of - Deconvolution layer with stride 2 , Concatenation with the corresponding cropped feature map from the contracting path and two $3 \times 3$ Convolution layer + activation function. The purpose of this expanding route is to allow for a specific position combined with the qualitative details of the contracting path.

\section{1. b. SEGNET:}

A CNN-based deep neural network proposed by Badrinarayanan et al. [26] for semantic segmentation in 2015. As seen in the diagram. 3, it consists of downsampling- upsampling blocks. The downsampling route consists of 5 encoders composed of 13 convolutional layers borrowed from the first 13 layers in the VGG16 network [27]. Each encoder layer has the corresponding decoder layer and thus the decoder route also has 13 layers. Each encoder allows a convolution with a filter set to construct a sequence of feature maps for the normalization of batch with rectified linear unit (ReLU) operations applied, followed by Max pooling with a 2 x 2 window and stride 2 . One key principle of SegNet is the use of max-pooling indices in decoders to do upsampling of low-resolution feature maps. Pooling-indices has the advantage of preserving high-frequency information in processed images with reduced training parameters in the decoder portion.

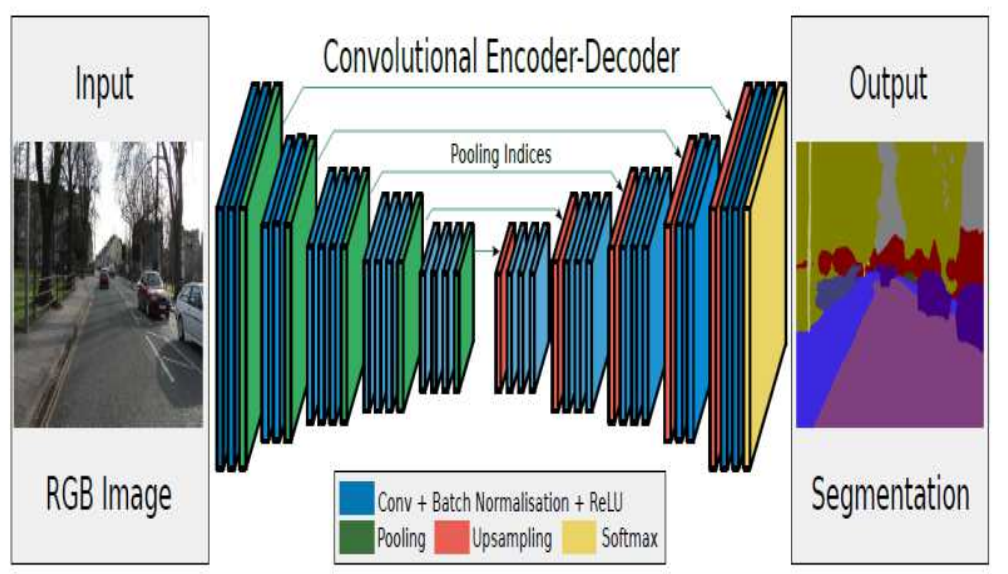

Fig. 3 SegNet architecture [26]

\subsection{Dataset Description}

We used publicly accessible brain tumor dataset-Figshare [28]-to train and test the UNET and SEGNET segmentation 
TABLE I

DETAIL OF UNET ARCHITECTURE. INPUT IMAGE SIZE IS 256 X 256X 3. FILTERS OF 3 X 3 SIZES ARE USED FOR EACH LAYER. HERE, BATCHNORM STANDS FOR BATCH NORMLIZATION, M STANDS FOR MAX-POOLING LAYER, AND U STANDS FOR UPSAMPLING LAYER.

\begin{tabular}{|c|c|c|c|c|c|c|}
\hline Block & Layer types & No. of Repetitions & No. of Filters & Layer Name, Stride & Input Size & Output Size \\
\hline Downsampling 1 & Convolution + BatchNorm + ReLU & 2 & 64 & M, 2 & $256,256,3$ & $128,128,64$ \\
\hline Downsampling 2 & Convolution + BatchNorm + ReLU & 2 & 128 & $\mathrm{M}, 2$ & $128,128,64$ & $64,64,128$ \\
\hline Downsampling 3 & Convolution + BatchNorm + ReLU & 2 & 256 & M, 2 & $64,64,128$ & $32,32,256$ \\
\hline Downsampling 4 & Convolution + BatchNorm + ReLU & 2 & 512 & M, 2 & $32,32,256$ & $16,16,512$ \\
\hline Upsampling 1 & Convolution + BatchNorm + ReLU & 2 & 1024 & $\mathrm{U}, 2$ & $16,16,512$ & $32,32,1024$ \\
\hline Upsampling 2 & Convolution + BatchNorm + ReLU & 2 & 512 & $\mathrm{U}, 2$ & $32,32,1024$ & $64,64,512$ \\
\hline Upsampling 3 & Convolution + BatchNorm + ReLU & 2 & 256 & $\mathrm{U}, 2$ & $64,64,512$ & $128,128,256$ \\
\hline Upsampling 4 & Convolution + BatchNorm + ReLU & 2 & 128 & $\mathrm{U}, 2$ & $128,128,256$ & $256,256,128$ \\
\hline Upsampling 5 & Convolution + BatchNorm + ReLU & 2 & 64 & -- & $256,256,128$ & $256,256,64$ \\
\hline Sigmoid Layer & & & & & $256,256,64$ & $256,256,1$ \\
\hline
\end{tabular}

TABLE II

DETAILS OF THE DESIGN OF SEgNET. THE RESOLUTION OF THE INPUT IMAGE IS 256 X 256X 3 . FILTERS OF 3 X 3 SIZES ARE USED FOR EACH LAYER. HERE, BATCHNORM STANDS FOR BATCH NORMALIZATION, M STANDS FOR MAX-POOLING LAYER, AND U STANDS FOR UPSAMPLING LAYER.

\begin{tabular}{|c|c|c|c|c|c|c|}
\hline Block & Layer types & No. of Repetitions & No. of Filters & Layer Name, Stride & Input Size & Output Size \\
\hline Encoder 1 & Convolution + BatchNorm + ReLU & 2 & 64 & $\mathrm{M}, 2$ & $256,256,3$ & $128,128,64$ \\
\hline Encoder 2 & Convolution + BatchNorm + ReLU & 2 & 128 & $\mathrm{M}, 2$ & $128,128,64$ & $64,64,128$ \\
\hline Encoder 3 & Convolution + BatchNorm + ReLU & 3 & 256 & $\mathrm{M}, 2$ & $64,64,128$ & $32,32,256$ \\
\hline Encoder 4 & Convolution + BatchNorm + ReLU & 3 & 512 & M, 2 & $32,32,256$ & $16,16,512$ \\
\hline Encoder 5 & Convolution + BatchNorm + ReLU & 3 & 512 & $\mathrm{M}, 2$ & $16,16,512$ & $8,8,512$ \\
\hline Decoder 1 & Convolution + BatchNorm + ReLU & 3 & 512 & $\mathrm{U}, 2$ & $8,8,512$ & $16,16,512$ \\
\hline Decoder 2 & Convolution + BatchNorm + ReLU & 3 & 256 & $\mathrm{U}, 2$ & $16,16,512$ & $32,32,512$ \\
\hline Decoder 3 & Convolution + BatchNorm + ReLU & 3 & 128 & $\mathrm{U}, 2$ & $32,32,512$ & $64,64,256$ \\
\hline Decoder 4 & Convolution + BatchNorm + ReLU & 2 & 64 & $\mathrm{U}, 2$ & $64,64,256$ & $128,128,128$ \\
\hline Decoder 5 & Convolution + BatchNorm + ReLU & 2 & 32 & $\mathrm{U}, 2$ & $128,128,128$ & $256,256,64$ \\
\hline Sigmoid Layer & & & & & $256,256,64$ & $256,256,1$ \\
\hline
\end{tabular}

and 613 images in images in test set. To avoid the overfitting

networks. The dataset contains 3064 brain MRIs obtained from 233 patients. It comprises three types of brain tumors: meningioma (708), pituitary (930), and glioma (1426). The data set is already available ".mat" format in MATLAB. The configuration of the ".mat" file comprising a patient ID, a unique mark indicating the type of brain tumor, $512 \times 512$ image data in uint16 format, a vector containing a tumor boundary with distinct point coordinates, and ground truth in a binary mask image.

\subsection{Preprocessing and Data Augmentation}

The images and ground truths extracted are reduced to size 256 X 256 to have low computational cost. The image data is considered in colored (RGB channels) whereas ground truths are in binary as shown in figure 4.
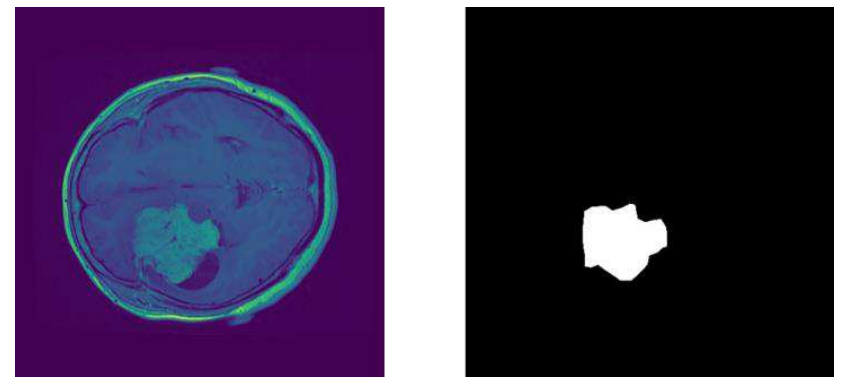

Fig. 4 Colored image and binary ground truth

We have divided 3064 images of dataset into $80 \%$ training data and $20 \%$ testing data. Thus 2451 images in training set
[29] and to have large training dataset, augmentation is performed. Training images in this work were augmented by the inclusion of knowledge inside the training images itself. In this technique the data augmentation methods like rescale, flip, rotation, zoom, shear, brightness etc. with various parameters are applied for creating new larger training set as shown in fig 5 . In this way we have increased training data set to 10 times of original size i.e., total training images are 24510 .

\section{EXPERIMENTATION AND RESULTS}

The implementation of UNET and SEGNET has been carried out with KERAS using TensorFlow as backend in python. The networks have been trained for number of epochs using NVDIA TESLA V100 graphics card with 16 GB of dedicated memory with 192 GB of DDR4, $2666 \mathrm{MHz}$ and Intel Xeon SKL G-6148 processor. Hyperparameters of the network, such as rate of learning, size of batch, number of epochs, are tuned on a trial-and-error basis. The training optimizer used is Adam [30] with an initial 0.0001 learning rate. Adam, an extension of the stochastic gradient descent (SGD) algorithm, preserves the learning rate for each network weight and adapts it independently as learning continues. We used the "ReduceLROnPlateau" Keras callback to adaptively minimize the learning rate when the parameter has stopped improving. Models also benefit from the reduction of the learning rate by a factor of 10 after learning has stagnated. This callback tracks the quantity 

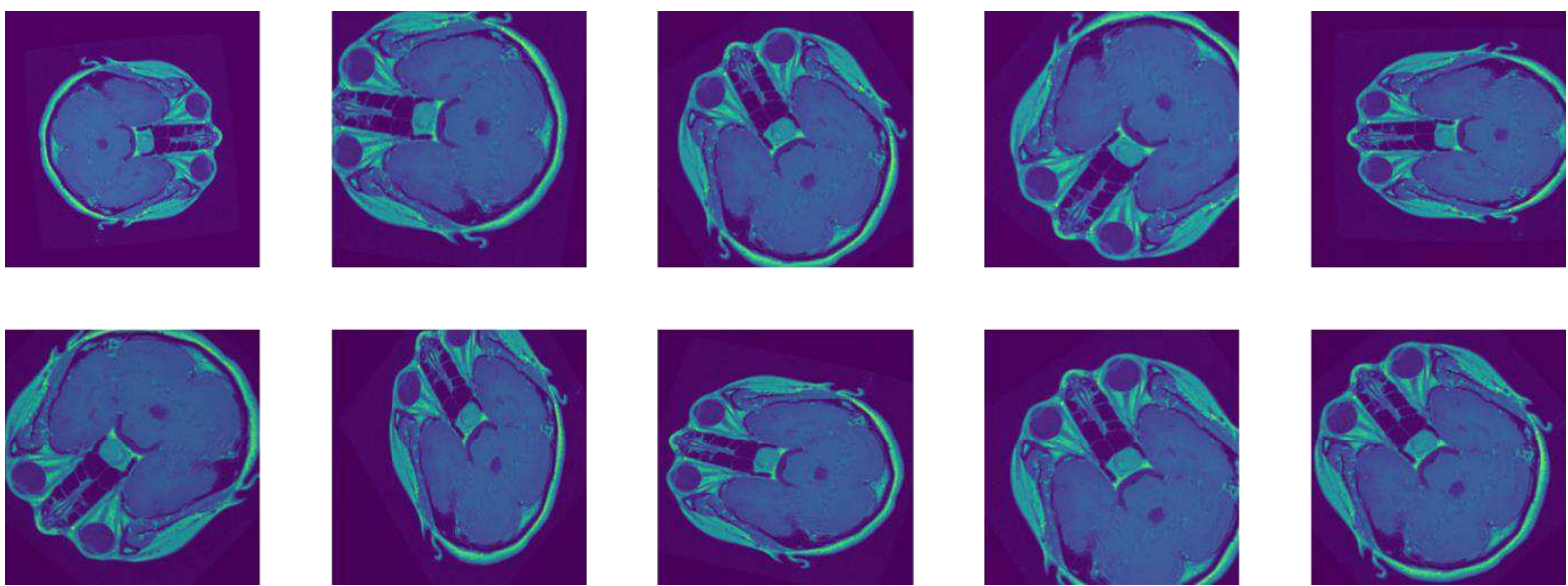

Fig. 5 Data augmentation result

and if there is no change in the "patience"-number of epochs, the learning rate is decreased.

\subsection{Evaluation metrics}

Following are the metrics to analyze the performance of segmentation networks:

I. Dice Similarity Coefficient: The statistic used to calculate the efficiency of the segmentation is the Dice similarity coefficient. It's the proportion of the intersection area to the union area between the ground truth image $(\mathrm{X})$ and the segmented image (Y) and can be found using the following equation-

$$
D(X ; Y)=\frac{2|X \cap Y|}{|X|+|Y|}
$$

II. Accuracy: Often known as classification accuracy, which is the ratio of accurate predictions to the total number of predictions made by the classifier. It can be shown by using the equation-

$$
\text { Accuracy }=\frac{T P+T N}{T P+T N+F P+F N}
$$

where, TP represents the number of true positive, i.e. pixels that are properly classified as tumor area, TN shows the number of real negative ones, i.e. pixels belonging to the background, properly classified as background, FP indicates the number of false positives, i.e. the number of wrongly classified as tumor pixels, and FN denotes the number of false negative, i.e. pixels belonging to tumor region falsely classified as background [31].

III. Precision: Percentage of positive instances out of the total predicted positive instances. Here denominator is the model prediction done as positive from the whole given dataset. Take it as to find out 'how much the model is right when it says it is right?'. It is used to find the actual tumorous pixels.

$$
\text { Precision }=\frac{T P}{T P+F P}
$$

IV. Loss Function: The function used to measure the loss is Binary cross entropy also called Sigmoid Cross-Entropy loss. The equation for Binary CrossEntropy loss as shown below-

$$
L\left(\mathrm{y}_{i}, \hat{\mathrm{y}}_{i}\right)=-\frac{1}{N} \sum_{i=0}^{N}\left(\left(\mathrm{y}_{i} * \log \left(\hat{\mathrm{y}}_{i}\right)+\left(1-\mathrm{y}_{i}\right) * \log \left(1-\hat{\mathrm{y}}_{i}\right)\right)\right)
$$

where $\hat{y}$ is the predicted value and y is actual value.

\subsection{Training performance}

The training performance of UNET and SEGNET is shown in figure 6 . The various training experiments with different hyper parameters are performed on both the networks. The best training performance with suitable final hyperparameters are shown in table 3.

TABLE III

TRAINING PARAMETERS

\begin{tabular}{ccc}
\hline Network Architecture & UNET & SEGNET \\
\hline Optimizer & \multicolumn{2}{c}{ Adam } \\
\hline Initial Learning Rate & 0.0001 \\
\hline No. of epochs & \multicolumn{2}{c}{235} \\
\hline Images & 24510 & 24510 \\
\hline Binary cross entropy loss & $\mathbf{0 . 0 1 7 9 5 9 9 6}$ & 0.021663887 \\
\hline Dice Similarity Coefficient & $\mathbf{0 . 8 0 0 0 9 6 5 1 2}$ & 0.701303184 \\
\hline Accuracy & $\mathbf{0 . 9 7 8 7 1 0 4 7 3}$ & 0.978591979 \\
\hline Precision & $\mathbf{0 . 9 5 1 3 6 2 9 0 8}$ & 0.921810567 \\
\hline
\end{tabular}


As depicted in figure $6 \mathrm{~b}$ loss of UNET is minimum than SEGNET. The training Dice Similarity Coefficient is raised to $\mathbf{0 . 8 0}$ and $\mathbf{0 . 7 0}$ for UNET and SEGNET respectively as shown in 6a. Fig 6c and 6d denotes the Accuracy and Precision of both the networks respectively. UNET performed well as compared to SEGNET in all respects.

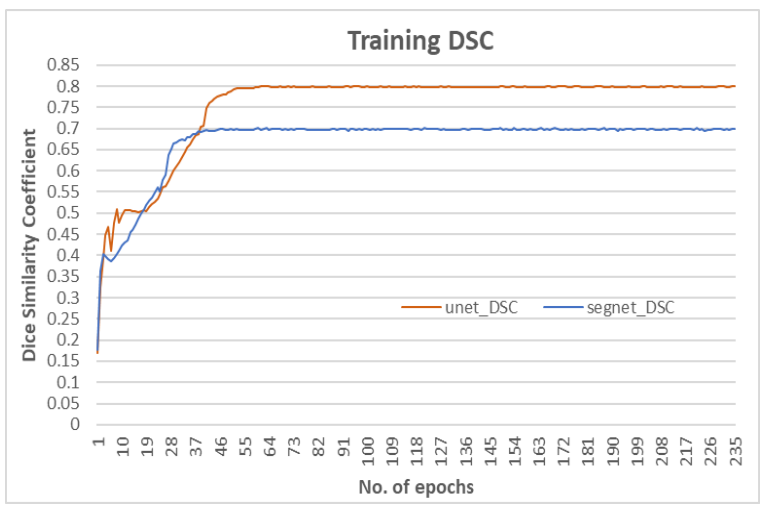

(a)

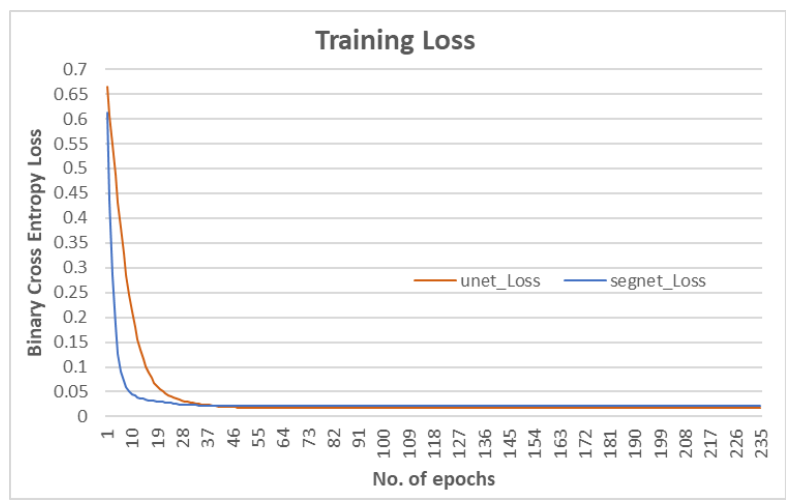

(b)

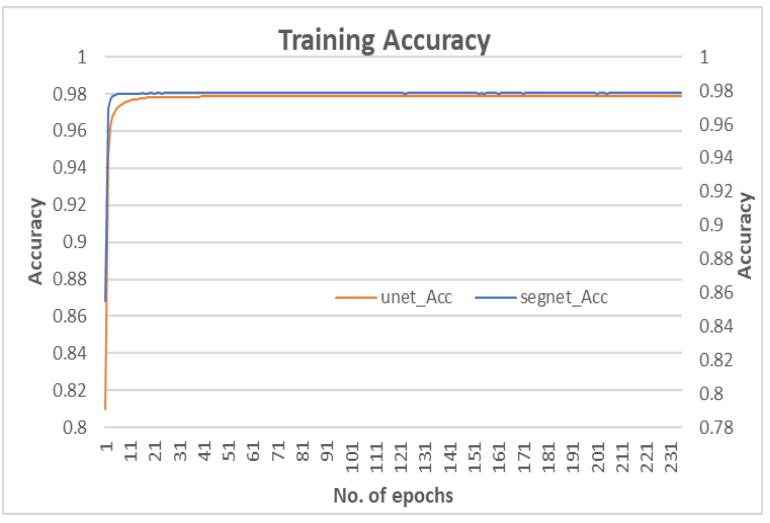

(c)

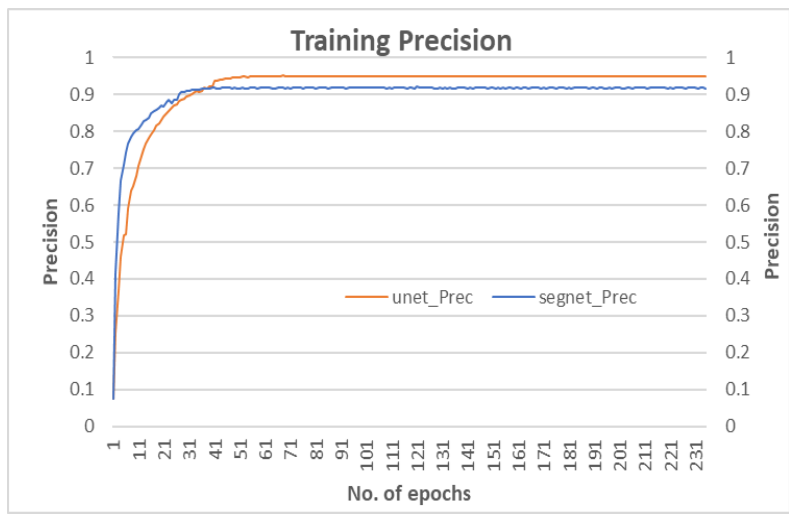

(d)

Fig. 6 Performance on Training Dataset a. DSC, b. Loss, c. Accuracy, d. Precision

\subsection{Segmentation Results}

The UNET and SEGNET networks are evaluated on Test dataset of size 613 images. The average DSC of testing dataset for UNET and SEGNET is 0.76 and 0.67 respectively with precision score of 0.90 for both. The accuracy and precision of the segmented image is often higher than the DSC, as it also involves the correctly classified background area. In addition, it should not rationally present the predictive performance of the model since it suffers from the contradiction of accuracy (which suggests that a trained model with a given degree of accuracy can have more predictive power than the models do better accuracy). Thus, when analyzing the segmentation networks, we put more emphasis on DSC in addition to accuracy and precision.

The visualization of predictions of some of the testing images segmentations are shown in figure 7. It is clearly indicated that UNET outperformed over the SEGNET.

\section{DISCUSSION AND CONCLUSION}

Segmenting Brain tumors is very challenging task still that is carried out by humans. However, there is demand for automatic segmentation which benefitted a lot that shown in deep learning research. In this paper, we have shown the effective use of semantic segmentation networks i.e. UNET and SEGNET in automatic brain tumor segmentation. We have shown that UNET has better segmentation results than SEGNET trained on figshare brain MRI image dataset. 


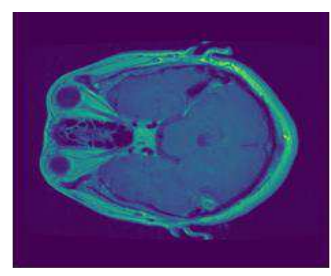

Test Image

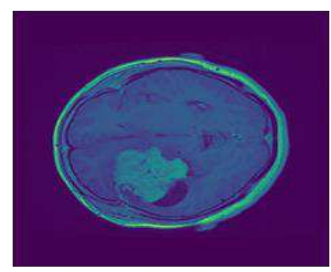

Test Image

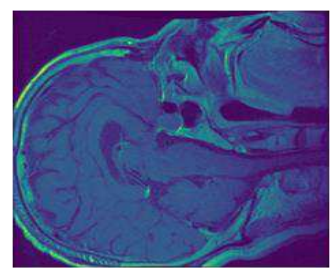

Test Image

Column (a)

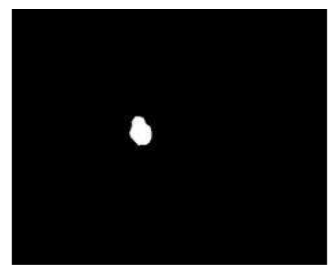

Ground Truth

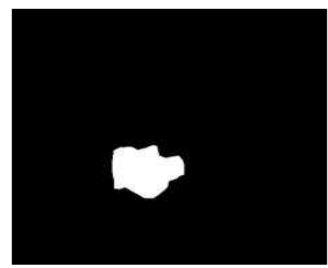

Ground Truth

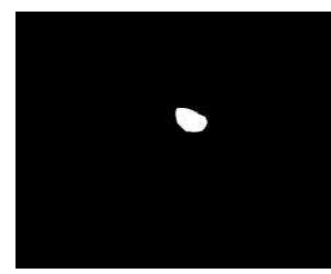

Ground Truth

Column (b)

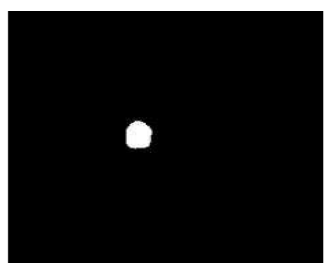

$\mathrm{DSC}=\mathbf{0 . 9 3}$

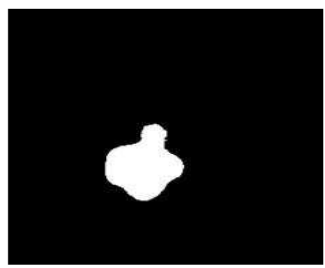

$\mathrm{DSC}=\mathbf{0 . 8 6}$

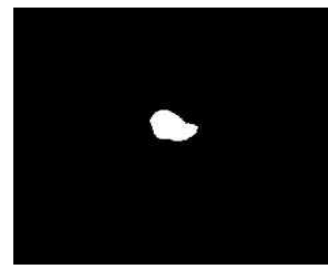

$\mathrm{DSC}=0.81$

Column (c) UNET Prediction

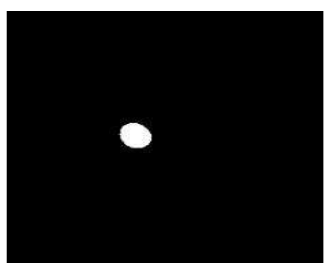

$\mathrm{DSC}=\mathbf{0 . 9 0}$

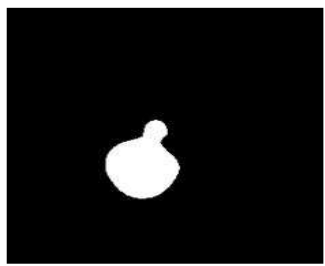

$\mathrm{DSC}=\mathbf{0 . 8 2}$

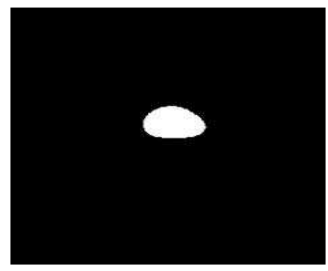

$\mathrm{DSC}=0.79$

Column (d) SEGNET Prediction

Fig. 7 Visualization of Segmented Results a. Test images, b. Ground Truths, c. Prediction by UNET d. Prediction by SEGNET

We aim to further improve the performance of both the networks by dividing whole image into number small patches in training dataset. In future, we should state the dice similarity coefficient separately for tumor and background to better analyze the segmented images. Also, effect of expanding encoder-decoder blocks in each network on segmentation results can be further analyzed. After successful segmentation with high DSC, we try automatically to classify the type of tumor in one of class as glioma, meningioma and pituitary tumors.

\section{Acknowledgement}

The support and the resources provided by 'PARAM Shakti Facility' under the National Supercomputing Mission, Government of India at the Indian Institute of Technology, Kharagpur are gratefully acknowledged. The authors are also grateful to the anonymous referees for the insights and the priceless recommendations provided in order to progress the initial version of this manuscript.

\section{Funding}

Financial support under the scheme 'Collaborative Research Innovation Project (CRIP)', TEQIP-III, Government of India through Dr. Babasaheb Ambedkar Technological University, Lonere Raigad, M.S. India is gratefully acknowledged.

\section{Conflict of Interest}

The authors declare that they have no conflict of interest.

\section{Data availability statement}

The dataset comes from the work of J. Cheng et al. [28] (https://doi.org/10.6084/m9.figshare.1512427.v5). contains three kinds of Brain tumor MRI Images: meningioma (708), pituitary (930), and glioma (1426), which are stored in four folders.

\section{Ethics approval and consent to participate}

This article uses publicly available data and therefore does not contain any studies with human participants or animals.

\section{Consent to participate}

Informed consent was obtained from all individual participants included in the study.

\section{Authors' contributions}


PEK, SMJ and VK have contributed to the research with the order they appear. PEK and VK are principal investigator and co-investigator of research project respectively. SMJ is research supervisor. Also, all authors have discussed the final results as well as improved the final manuscript.

\section{REFERENCES}

[1] A. de Brebisson and G. Montana, "Deep neural networks for anatomical brain segmentation," in CVPR Workshops, 2015, pp. $20-28$.

[2] M. Avendi, A. Kheradvar, and H. Jafarkhani, "A combined deeplearning and deformable-model approach to fully automatic segmentation of the left ventricle in cardiac MRI," Medical Image Analysis, vol. 30, pp. 108-119, 2016.

[3] H. R. Roth, H. Oda, Y. Hayashi, M. Oda, N. Shimizu, M. Fujiwara,K. Misawa, and K. Mori, "Hierarchical 3D fully convolutional networks for multi-organ segmentation," arXiv preprint:1704.06382, 2017.

[4] O. Ronneberger, P. Fischer, and T. Brox, "U-net: Convolutional networks for biomedical image segmentation," in MICCAI, 2015, pp. $234-241$.

[5] I. Razzak, M. Imran, G. Xu, Efficient brain tumor segmentation with multi scaleancer statistics two pathway- group conventional neural networks. IEEE J. Biomed. Health Inf. (2018). https://doi.org/10.1109/JBHI.2018.2874033

[6] N. Abiwinanda, M.Hanif, S.T.Hesaputra, A.Handayani, T.R.Mengko, Brain tumor classification using convolutional neural network. In World Congress on Medical Physics and Biomedical Engineering, pp. 183-189. Springer (2019)

[7] T.A. Abir, J.A. Siraji, E. Ahmed, B. Khulna, Analysis of a novel MRI based brain tumour classification using probabilistic neural network (PNN). Int. J. Sci. Res. Sci. Eng. Technol. 4(8), 65-79 (2018)

[8] Arshia Rehman1 - Saeeda Naz1 - Muhammad Imran Razzak2 ·Faiza Akram3 - Muhammad Imran4A Deep Learning-Based Framework for Automatic Brain Tumors Classification Using Transfer Learning, Circuits, Systems, and Signal Processing https://doi.org/10.1007/s00034-019-01246-3

[9] Yao J. Image processing in tumor imaging. New techniques in oncologic imaging; 2006. p. 79-102.

[10] Sergio Pereira, Adriano Pinto, "Brain Tumor Segmentation Using Convolutional Neural Networks in MRI Images", IEEE TRANSACTIONS ON MEDICAL IMAGING, VOL. 35, NO. 5, MAY 2016

[11] Dong H, Yang G, Liu F, Mo Y, Guo Y (2017). Automatic brain tumor detection and segmentation using U-net based fully convolutional networks. MIUA, 3, 1-12.

[12] Mohammad Havaei, (2016) "Brain Tumor Segmentation with Deep Neural Networks", Preprint submitted to Medical Image Analysis

[13] Saddam Hussain, Syed Muhammad Anwar, "Brain Tumor Segmentation using Cascaded Deep Convolutional Neural Network", 978-1-5090-2809-2/17/\$31.00 (O2017 IEEE

[14] Wang G, Li W, Azuluaga M, et al (2018). Interactive medical image segmentation using deep learning with image specific fine tuning. IEEE Trans Med Imaging, 37, 1562- 73

[15] Konstantinos KK, Ledig C, Virginia, FJ, et al (2017). Efficient multi scale 3D CNN with fully connected CRF for accurate brain lesion segmentation. Med Image Analysis, 36, 61-78

[16] Liang Chen, Paul Bentley, "DRINet for Medical Image Segmentation", DOI 10.1109/TMI.2018.2835303, IEEE Transactions on Medical Imaging
[17] Dmitry Lachinov, "Glioma Segmentation with Cascaded Unet", arXiv: $1810.04008 \mathrm{v} 1$ [cs.CV] 9 Oct 2018

[18] Cui S, Mao L, Jiang J, Liu C, Xiong S (2018). Automatic semantic segmentation of brain gliomas from MRI images using a deep cascaded neural network. J Healthcare Engineering, 2018, 1-14.

[19] Le H.T., Pham H.TT. (2020) Brain Tumor Segmentation Using U-Net Based Deep Neural Networks. In: Van Toi V., Le T., Ngo H., Nguyen TH. (eds) 7th International Conference on the Development of Biomedical Engineering in Vietnam (BME7). BME 2018. IFMBE Proceedings, vol 69. Springer, Singapore. https://doi.org/10.1007/978981-13-5859-3_7

[20] Ben Naceur M., Akil M., Saouli R., Kachouri R. (2020) Deep Convolutional Neural Networks for Brain Tumor Segmentation: Boosting Performance Using Deep Transfer Learning: Preliminary Results. In: Crimi A., Bakas S. (eds) Brainlesion: Glioma, Multiple Sclerosis, Stroke and Traumatic Brain Injuries. BrainLes 2019. Lecture Notes in Computer Science, vol 11993. Springer, Cham. https://doi.org/10.1007/978-3-030-46643-5_30

[21] Krizhevsky, A., Sutskever, I., \& Hinton, G. E. (2012). Imagenet classification with deep convolutional neural networks. In Advances in neural information processing systems, pp. 1097-1105.

[22] Simonyan, K., \& Zisserman, A. (2014). Very deep convolutional networks for large-scale image recognition. In Proceedings of international conference on learning representations. arXiv:1409.1556

[23] Szegedy, C., Liu, W., Jia, Y., Sermanet, P., Reed, S., Anguelov, D., Erhan, D., Vanhoucke, V., \& Rabinovich, A. (2015). Going deeper with convolutions. In Computer vision and pattern recognition (CVPR). arXiv:1409.4842

[24] Ronneberger, O., Fischer, P., \& Brox, T. (2015). U-net: Convolutional networks for biomedical image segmentation. In International conference on medical image computing and computer-assisted intervention (pp. 234-241). New York: Springer.

[25] Long, J., Shelhamer, E., Darrell, T.: Fully convolutional networks for semantic segmentation (2014), arXiv:1411.4038 [cs.CV]

[26] Badrinarayanan, V., Kendall, A., \& Cipolla, R. (2015). Segnet: A deep convolutional encoder-decoder architecture for image segmentation. arXiv preprint arXiv:1511.00561

[27] Simonyan, K., \& Zisserman, A. (2014). Very deep convolutional networks for large-scale image recognition. In Proceedings of international conference on learning representations. arXiv:1409.1556

[28] J. Cheng, Brain tumor dataset. figshare. dataset. https://doi.org/10.6084/m9.figshare.1512427.v5. Accessed 30 May 2018

[29] Wang, J., \& Perez, L. (2017). The effectiveness of data augmentation in image classification using deep learning. Tech. rep., Technical report.

[30] Kingma, D., \& Ba, J. (2015). Adam: A method for stochastic optimization. In Proceedings of the 3rd international conference on learning representations (ICLR).

[31] Ajay Mittal, Rahul Hooda, Sanjeev Sofat, "LF-SegNet: A Fully Convolutional Encoder-Decoder Network for Segmenting Lung Fields from Chest Radiographs", Wireless Personal Communications, Issue $1 / 2018$. 
Figures

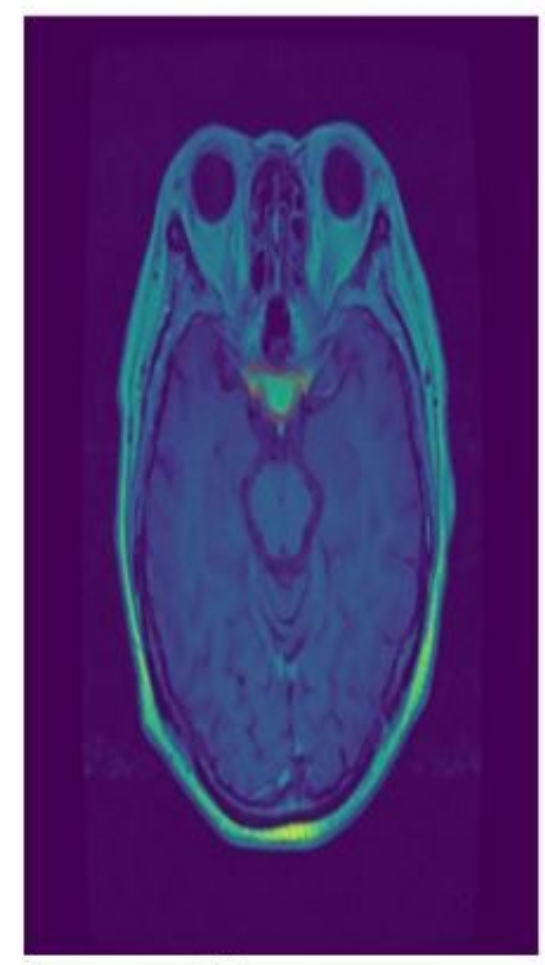

(a)

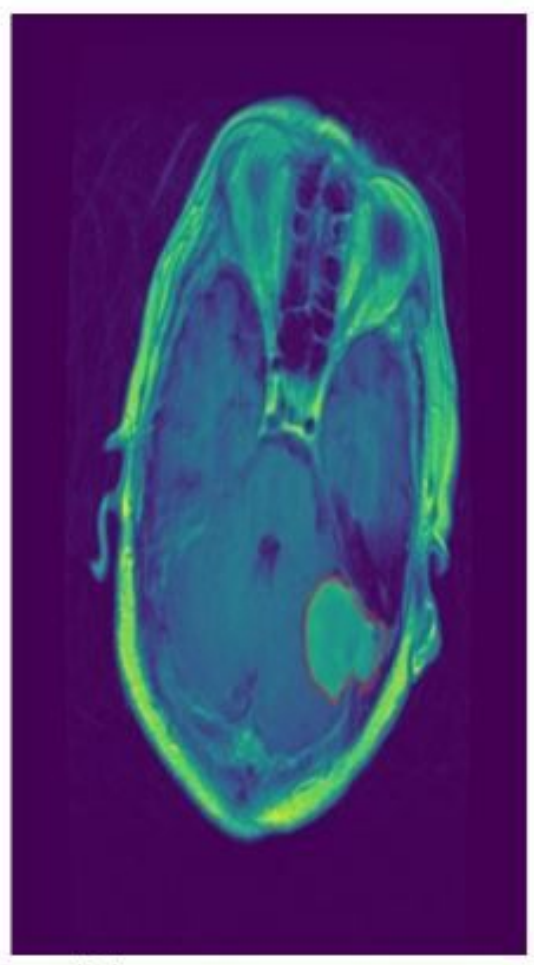

(b)

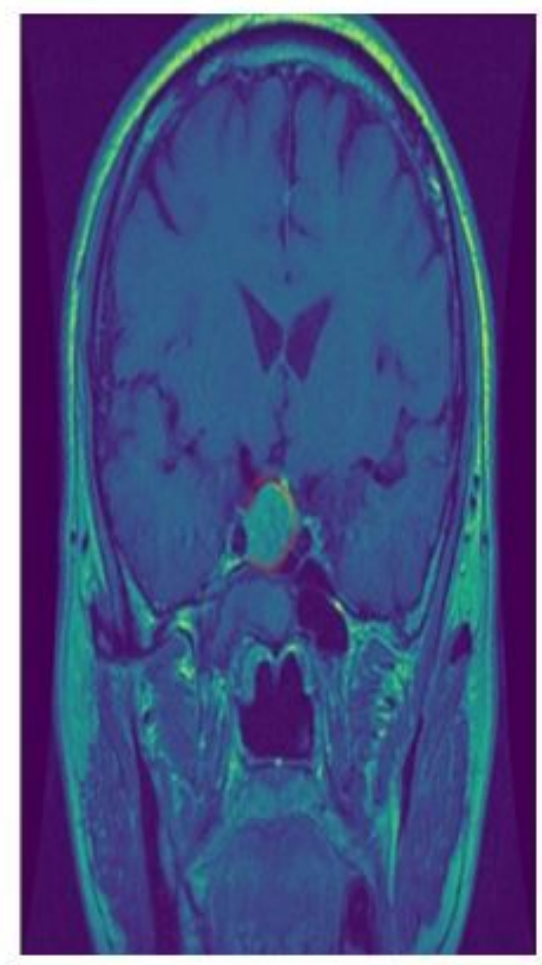

(c)

\section{Figure 1}

Illustrations of three typical brain tumors: (a) meningioma; (b) glioma; and (c) pituitary tumor. Red lines indicate the tumor border 


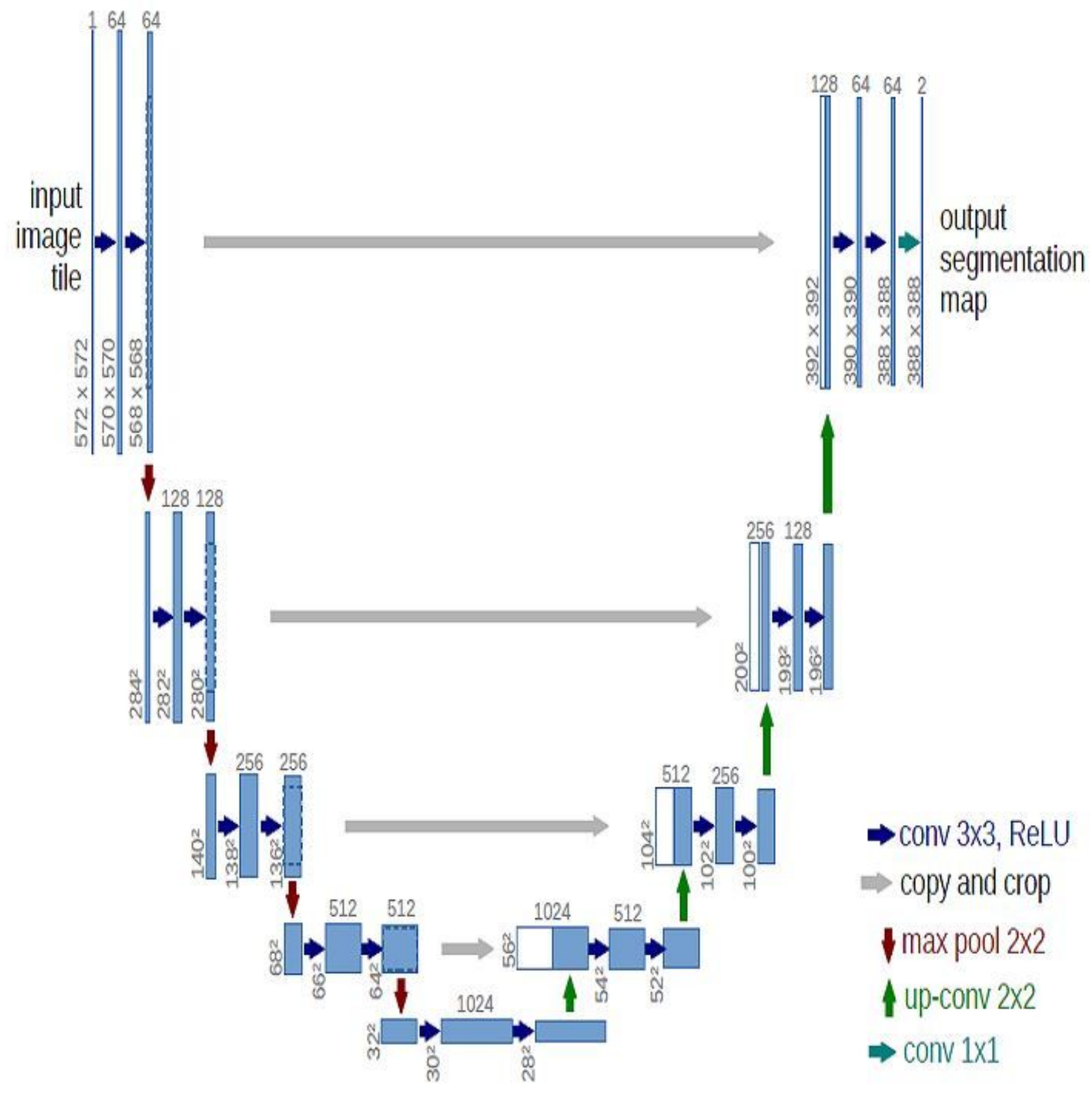

Figure 2

The architecture of the U-net. The boxes correspond to a map of a multi-channel function. The number of channels is displayed at the top of the box. The $x$-y-size is seen at the bottom left edge of the box. White boxes reflect copied feature maps. The arrows signify the various operations [24]. 


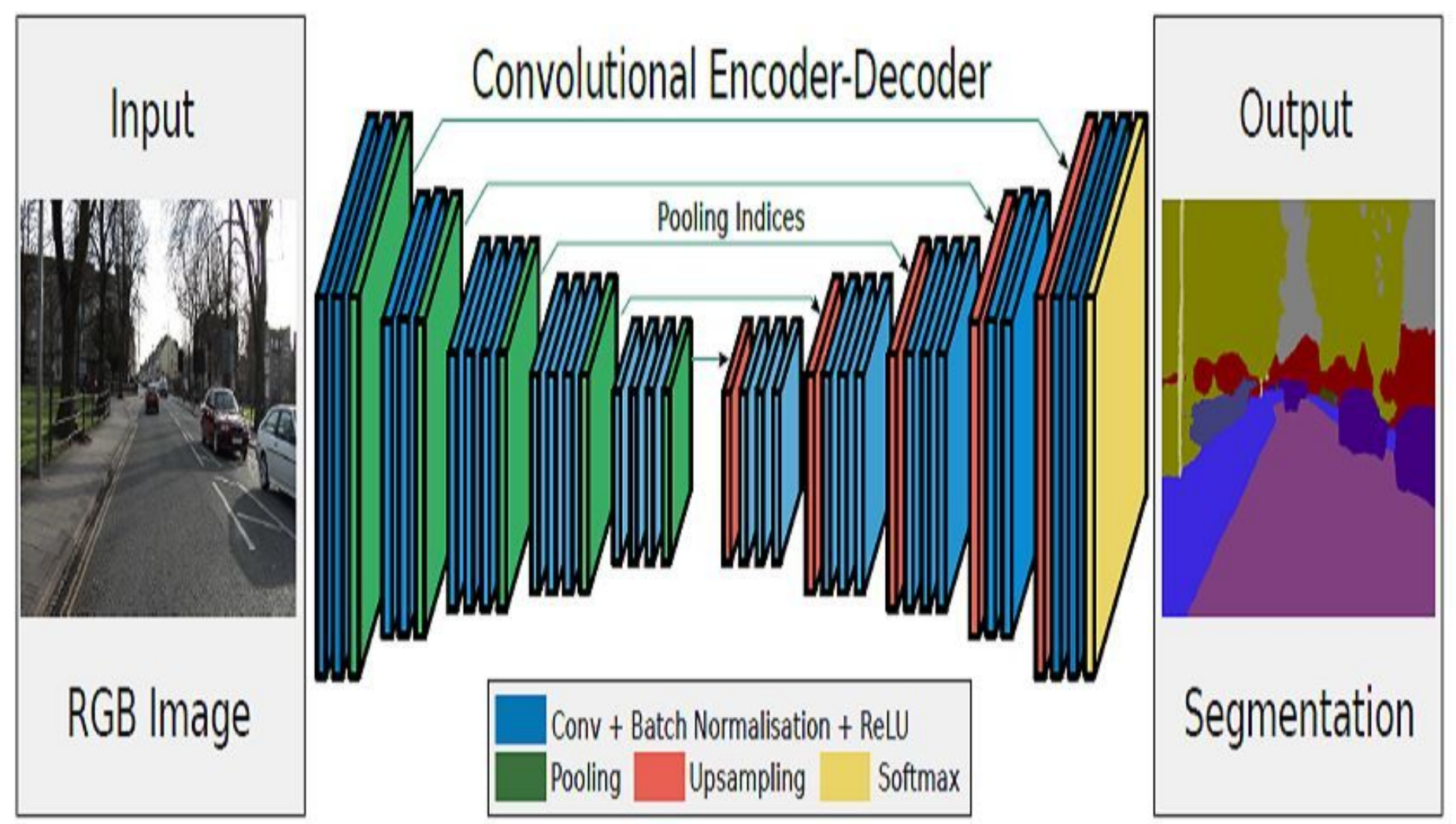

Figure 3

SegNet architecture [26]
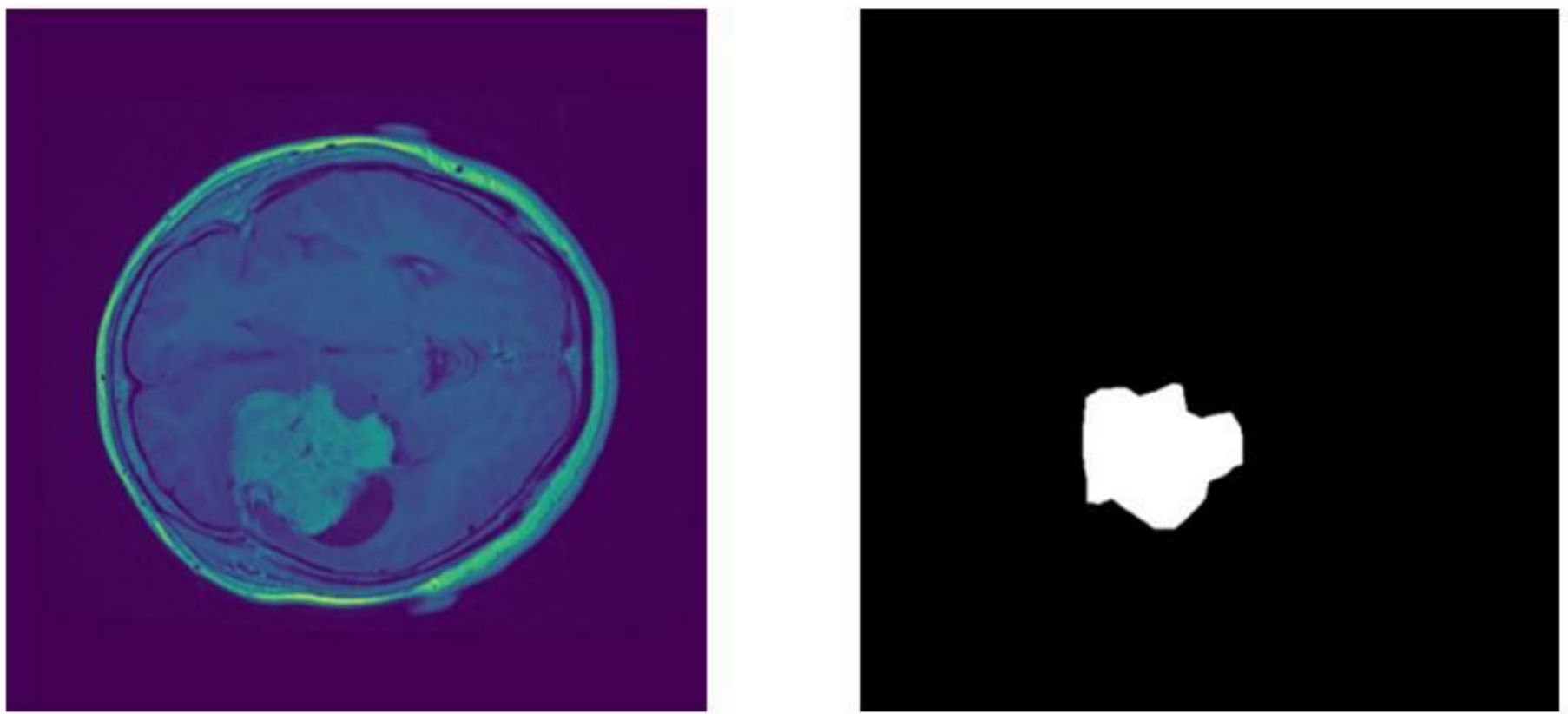

Figure 4 
Colored image and binary ground truth
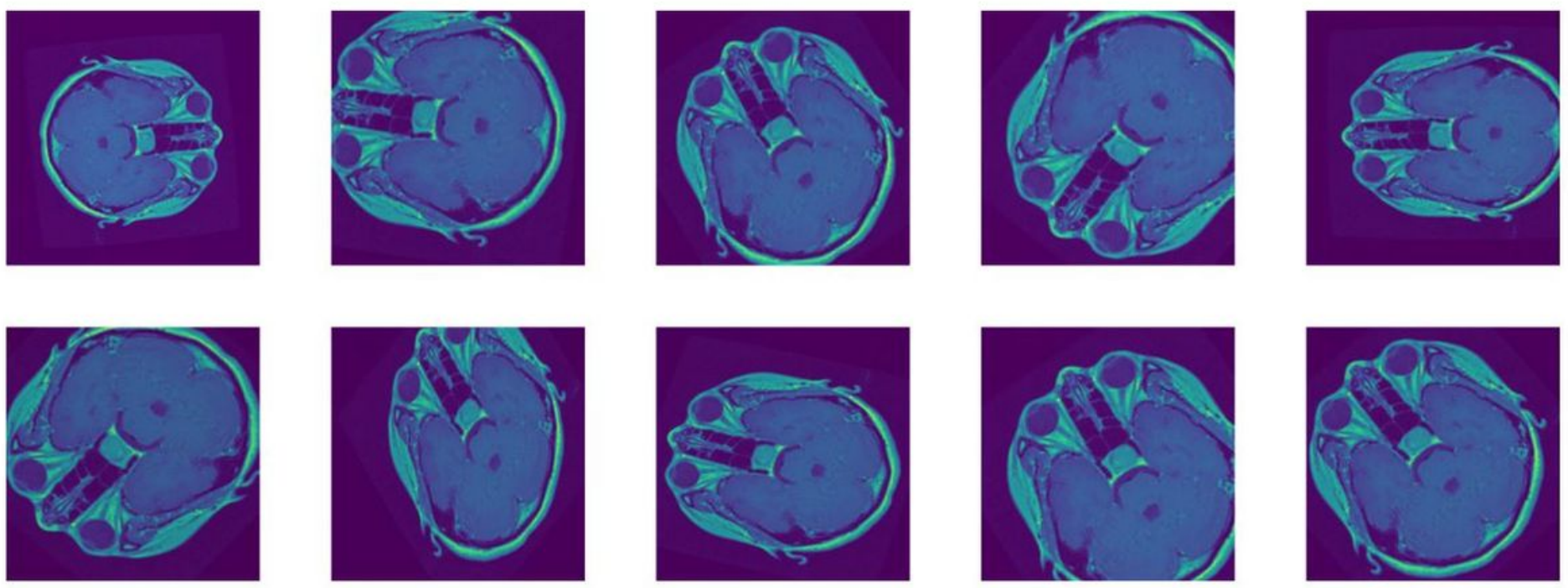

Figure 5

Data augmentation result 


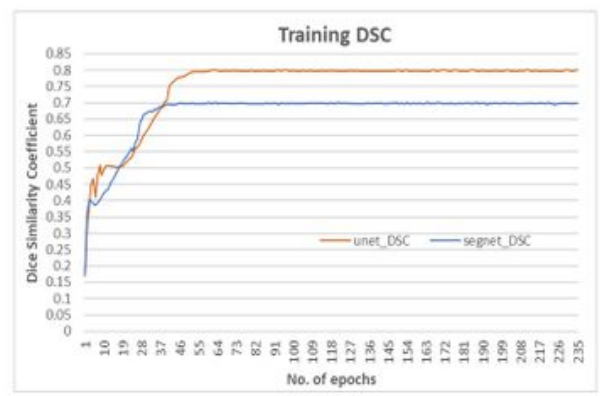

(a)

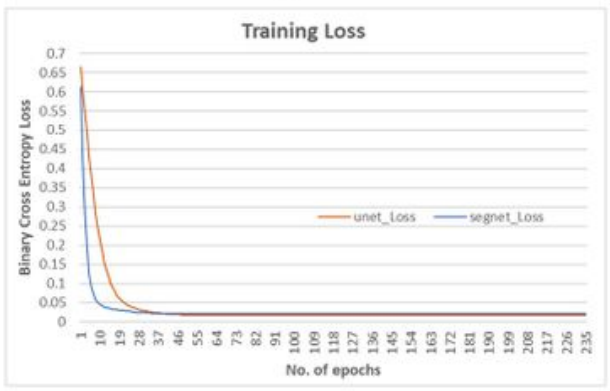

(b)

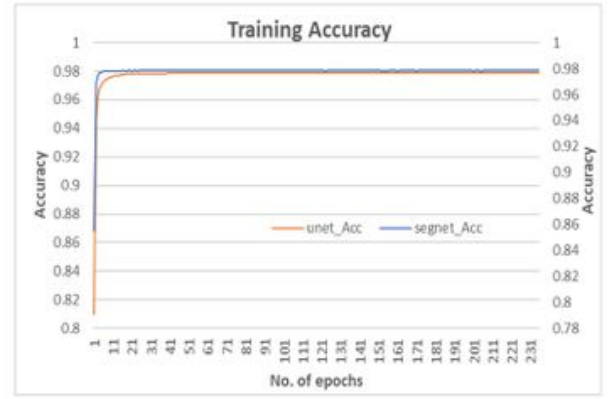

(c)

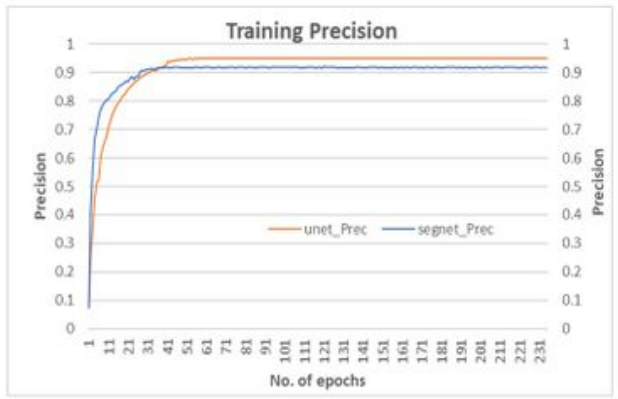

(d)

Figure 6

Performance on Training Dataset a. DSC, b. Loss, c. Accuracy, d. Precision 


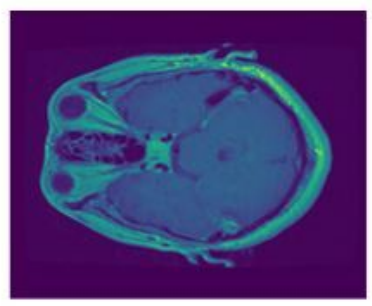

Test Image

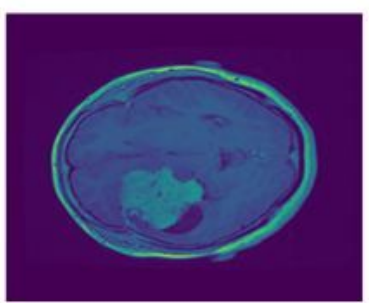

Test Image

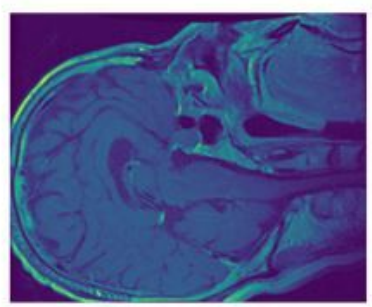

Test Image

Column (a)

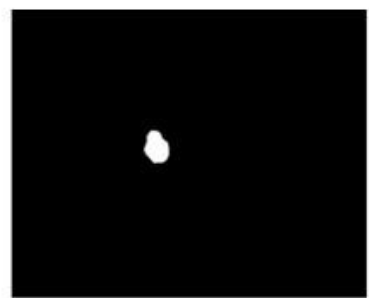

Ground Truth

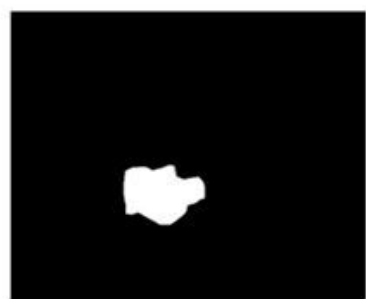

Ground Truth

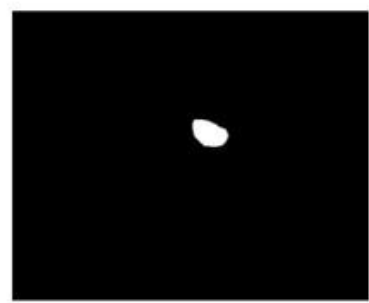

Ground Truth

Column (b)

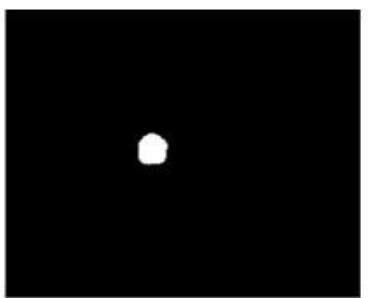

$\mathrm{DSC}=0.93$

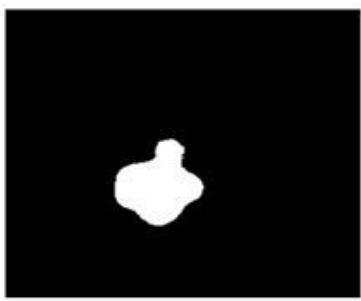

$\mathrm{DSC}=\mathbf{0 . 8 6}$

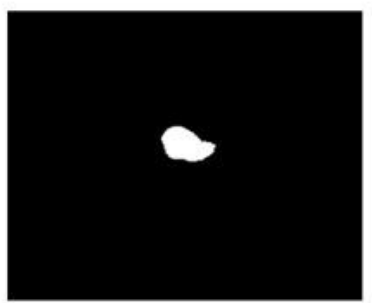

$\mathrm{DSC}=\mathbf{0 . 8 1}$

Column (c) UNET Prediction

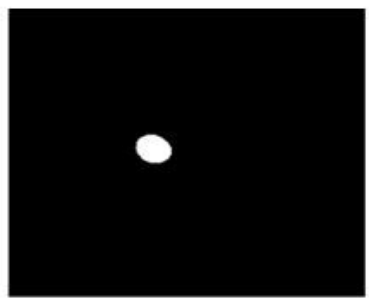

$\mathrm{DSC}=\mathbf{0 . 9 0}$

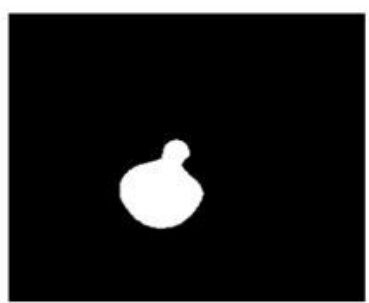

$\mathrm{DSC}=\mathbf{0 . 8 2}$

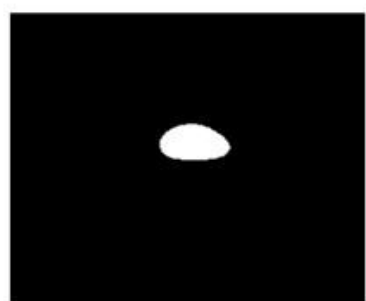

$\mathrm{DSC}=\mathbf{0 . 7 9}$

Column (d) SEGNET Prediction

Figure 7

Visualization of Segmented Results a. Test images, b. Ground Truths, c. Prediction by UNET d. Prediction by SEGNET 